\title{
Cost-Effective Disinfection Robot Using UVC Radiation
}

\author{
Mansi Dhikle, Vinaya Dharne, Pankaja Gaikar, Kausar Fakir \\ Department of Electronics and Telecommunication, Ramrao Adik Institute of Technology, Navi Mumbai, India \\ mansidhikle@gmail.com, vinayadharne20@gmail.com, piyagaikar1999@gmail.com, kausarmf@gmail.com
}

\begin{abstract}
Sanitization with human efforts is not an easy task. Chances of contracting infections increases which leads to additional spread of bacteria. Currently, normal cleaning robots are used in most of the places but looking at the current situation the sanitization techniques need to be improved. The robot uses radiation of $\mathrm{UV}$ rays to kill the microrganisms. It gives a live video streaming of its surrounding using a Wi-fi based camera. With the help of Bluetooth module and android mobile, we can control the movement of the robot inside the room without being physically present. It is built with PIC Microcontroller and Ultraviolet-C (UVC) Sanitization LED. UV-C has bandwidth range of $200-280 \mathrm{~nm}$ and is most powerful when it comes to killing pathogens in the room. This allows us to sterilise the room effectively. By killing the germs, the UV light restricts their multiplication by destroying their reproductive system. Thus use of this robot lowers the threat of infection, cost of traditional cleaning and sterilisation and increases security in medical facilities. Thus, we are trying to implement a more efficient way of sanitization by building a Low cost UV sanitization Robot which can be used in small clinics and for household purpose.

Index Terms-UVC Sanitization LED, PIC Micrcontroller, Bluetooth module
\end{abstract}

\section{INTRODUCTION}

The Covid-19 pandemic due to the novel coronavirus SARS-CoV-2 has led to more than 2.5 million deaths and has infected more than 103 million people worldwide, which makes us rethink how organizations and societies can work with minimum or no physical contact. The economic and social loss caused by the epidemic are horrific: Millions of people at risk of falling into extreme poverty. Adherence to safety and work to create and promising them decent work and protection of employees rights in all industries is important. The actions aimed at saving lives and livelihoods should include expanding social protection with regard to the provision of universal health care and income support to the most respected.

Every day health workers are fighting to help control the spread of virus as it has infected many lives all over the world. Robots were also used to treat and provide support for isolated patients. The World Health Organization recommends physical isolation from nearby people world to prevent the transfer of Covid-19 public standards.

Cleaning and disinfection are very critical steps in stopping the spread of contagious diseases inside closed doors . However, due to a shortage of manpower, the persistent cleaning of indoor spaces has become a critical issue. Also, involving manpower carries a high threat of infection while working for a long time in such areas.

Sanitization in the high exposure areas of the virus, that are hospitals and medical wards, is indeed challenging and requires very high measures to be taken. But in spite of all these high-end measures taken, there is always a risk associated with it.[7]

Even though the virus is relatively fragile, it continues to hold on to both porous and non-porous surfaces for hours, This leads to increase in the chance of spreading and risk of infection. The lipids that coat the genetic cloth of the virus is easy to disrupt, and therefore sanitising surfaces can certainly destroy the virus.

Thus, mobile robots are considered as a feasible option to the issues associated with conventional cleaning and disinfecting techniques because of their validated capacity to assist humans in various software regions together with hospitals, aged homes, and industries.

The objective of this project is to create a robotic controlled vehicle which is designed to sanitize using UV light and control the operation through cell phone. The robot provides live streaming of the surrounding area.With easy algorithm and program, the robot is able to cover big ground areas[5]. Here, the use of robots reduces human contact with the bacteria.Use of this robotic, lowers the risk of contamination, cost of traditional cleansing and disinfection and most importantly acquires self belief and safety in medical facilities.

\section{Literature ReVieW}

Robots have wide use in Health Care Areas. These bots use drifting machines mounted with ipads to produce doctor tele-presence,drones for transportation of important or different medical instrumentation. Socially conscious bots utilized in the supervision of the older or physically/mentally restricted individual, and industrial robots like those want to sterilize patient areas or offer delivery.

A few bots are presently within the procedure of evaluation and development. There is a lot to recognize about the usage 
of robots in nearby settings. The comfort of robots can rely upon many variables, such a person, culture, a particular software, or a business.

To clear up the clinic disinfection disadvantage, many style requirements had to be enforced. There's a of lot of design requirements for a robotic disinfector and more if the robot is to be operated in a clinic.

A few analysis papers associated with medical reports are been studied and they show the influence on the management of good health professional.

The paper by Pacharawam Chanprakon[1] of "An Ultra-violet sterilization mechanism for disinfection" has developed a ultraviolet radiation mechanism for sterilization in an operative or a patient room. Their ultraviolet radiation larva has three $19.3 \mathrm{~W}$ of ultraviolet radiation LEDs mounted on the radiation bot platform covering $360^{\circ}$ direction.

The paper by Himadri Nath Saha[2] of "Iot Based Garbage Monitoring and Clearance Alert System" proposed IoT based smart garbage monitoring and clearance alert system in which RGB lights attached to the bin indicates the garbage level of the bin. An android app was also developed to send alerts from the microcontroller to the system.

The paper by Noriyuki Yagi[3] of "Sterilization Using $365 \mathrm{Nm}$ Uv-Led" has proposed Sterilization using 365nm UV-LED. This paper surveys the sterilization results of UV-led and proves that it is able to destroy the harmful bacteria. This paper suggests that UV-led is smaller and brighter than low-pressure mercury lamp and can be used for the motive of disinfection effectively.

The paper by T.Sivaranjani[4] of "Design Of a Portable Assistive Device for Disinfection Of Floor Using Bluetooth Technology" has proposed a multilevel planning for cleaning tasks to be performed by lowcost floor cleaners. This paper helps efficient ground cleaning device with sweeping, suction and mopping Operations. In addition to the cleaning, a UV Germicidal lamp has been used to kill the micro organisms present on the floor.

The paper by G Tuangzhi Dai[5] of "Design on measurement and control system of cleaning robot based on sensor array detection" has proposed use of grid scanning algorithm based on electric map realize floor coverage task, and designed a synthesis detection system based on sensor array finding method technology according to algorithm characteristics, experimental outcomes for obstacle detection by static finding indicate that the designed detection device improves cleaning robotic's surroundings notion and route search capability significantly.
The paper by Sahil Parmar[6] of "A Design Of Hybrid Floor UV-C Sterilizer Robot" has developed a Hybrid Floor UV-C sterilizer Robot, a fluid less device which can be used to perform the disinfection using UVC light and has a hidden vaccum device cleaner and a built in UPS system. The robot is cost-effective and user friendly.

The paper by Apeksha Wadibhasme[7] of "Sanitization Robot" has designed a wise medical assistant mechanism by exploring numerous contactless device technologies and is compact for economical management and induce a real time environment identification technology for its movement in an exceedingly clinic area.

The paper by Thomas Rubaek[8] of "Evaluation Of The Uv-Disinfection Robot" has developed a UV-Disinfection robot to decrease the spread of diseases and Hospital Acquired Infections (HAIs). This robotic is used to disinfect locations in health facility and other environments. This robot is designed as an addition to the existing cleaning cycle. The test outcomes of the designed Robot are that the robotic is able to damage extraordinary types of micro organisms.

\section{Proposed System}

The paper being presented here is a cost effective, portable UV Sanitization Robot. It is capable of sterilising the room or small objects without being physically present in the room. The major outcome of this robot is to support healthcare workers by reducing their exposure to bacteria as much as possible.

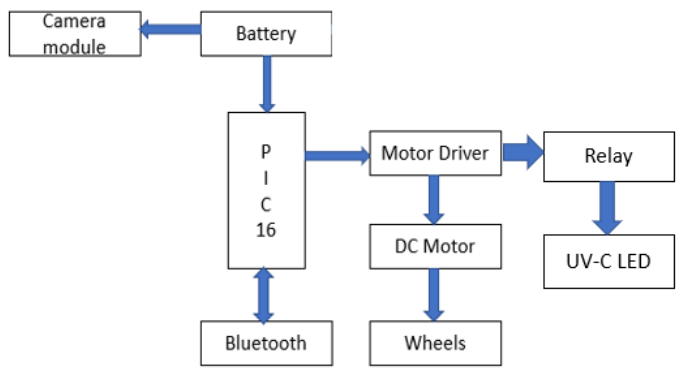

Fig. 1. Block Diagram of Proposed System

The Fig 1 shows the basic block diagram of the UV Sanitization Robot. This project uses Bluetooth decoder module and an andriod app to control the motion of the robot as well as to switch the UVC leds on and off.

The PIC microcontroller performs main actions like receiving data from the bluetooth module through serial communication and controls the motors based on the received 
data. The HC-05 Bluetooth module is interfaced with PIC microcontroller through serial communication.

\section{HARDWARE DESCRIPTION}

\section{A. PIC 16F877A Micrcontroller:}

The microcontroller plays a significant role in controlling the autonomous device. The main advantage of PIC is that it has a flash memory technology. An EEPROM is additionally present that allows the storage of data like transmitter codes and receiver frequencies and a few different needed data permanently. The cost of the controller is less and its use is simple. They are reliable and out of whack of PIC proportion is incredibly less. And performance of the PIC is incredibly quick due to exploitation computer architecture. Power consumption is also less compared to different microcontrollers. The price of this controller is less and its hosting is also easy [4]. It has wide range of applications and can be used in areas of microprocessor applications and timer functions

1)Instructions set - 35 .

2) Operational frequency- $20 \mathrm{MHz}$

3) Operating Voltage range $-4.2 \mathrm{~V}$ to $5.5 \mathrm{~V}$.

\section{B. HC-05 Bluetooth Decoder:}

It provides ASCII code output. This receiver enables wireless serial data transmission. It has a distance of 10 meters. Android phone and Bluetooth decoder attached on robot is used to control the robot The decoder is always connected to the Robot and the cell phone is used to control Robot movement.

The bluetooth decoder installed in the robot should be connected with Android phone. Every cell phone key is assigned a specific task. After connection, if the button is pressed, the corresponding button code is transmitted to the bluetooth decoder. With the help of Bluetooth decoder, the microcontroller will process the received code.

The android app used is HC-05 App which is available on any android mobile. Each key of the mobile is assigned a particular job.

\section{L293D Motor Driver:}

It is an electronic device that allows voltage to be applied across a load in both direction. It is used to convert $5 \mathrm{~V}$ to $12 \mathrm{~V}$ which is required to drive the motor and has an operational voltage of $5 \mathrm{~V}$. It is an electronic circuit which enables a voltage to be applied across a load in either direction.

\section{DC Motors:}

The motor uses power to provide energy, usually through magnetic field and current carrying coils. The DC motor generates power from the DC power provided by motor by converting DC to AC inside motor, constant magnetic field and rotating magnets.

\section{E. UVC LED:}

UVC leds are used as they are powerful and effetive in destroying viruses and other bacterial substances present in air and moisture [2]. This LED uses ultraviolet germicidal irradiation for killing the virus. Its operational voltage is $12 \mathrm{~V}$ and wavelength is of $270 \mathrm{~nm}$. The radiation kills the RNA in the virus and thus reduces the chance of transmission. From decades, the radiation are efficiently used for limiting the spread of bacteria. So UV-C lights are also known as "germicidal" lights.

\section{F. Power Supply:}

The battery is an integral part of the transmission module. The battery is used to produce $5 \mathrm{~V}$ and $12 \mathrm{~V}$ for DC supply ie $12 / 500 \mathrm{~mA}$ volts. Battery has output voltage of $12 \mathrm{~V}$ and current rating $=500 \mathrm{~mA}$

\section{G. Voltage Regulator:}

Two different power regulators 7805 and 7812 are used behind the capacitor filter to produce a permanent DC power supply of 5 volts and 12 volts. Both are three pin ICs namely input, ground and output.

\section{H. V380- Wifi based Camera:}

The camera is used for the live video streaming of the surrounding. It can be remotely controlled from a smartphone. The camera's resolution of 720 Pixel helps to obtain a clear view of the surrounding and rotates in 360 degree to give a direct view of every corner of the room. IR LEDs with ICR present in the cameras offers clear night vision for viewing and recording.

\section{Relay:}

Relays are electromagnetic switches which help to convert small electrical cuurents into very large currents and is used to provide isolation between the UV LED and the microcontroller.

\section{WORKING}

- The robot is provided with an android control which uses bluetooth communication.

- The android app can be used to control the device i.e. to move forward, right, left or reverse and to change the status of UV LEDs.

- The HC-05 Bluetooth module receives the input signal and sends it to the microcontroller.

- The microcontroller then executes these commands and sends it to the motor driver IC to drive the motor.

- The controlling of the UV Led's is done through the motor driver IC.

- The output pins of the motor driver ic is connected to the relay and then to the UV LEDs. 


\section{SOFTWARES USED}

- Eagle PCB design Software- EAGLE stands for Easily Applicable Graphical Layout Editor. It provides accurate solutions of pcb design and hence was used for designing of pcb.

- KEIL Microcontroller Software - This software was used to program the PIC microcontroller IC for movement of the robot. The programming was done in Assembly Language.

\section{FLOWCHART}

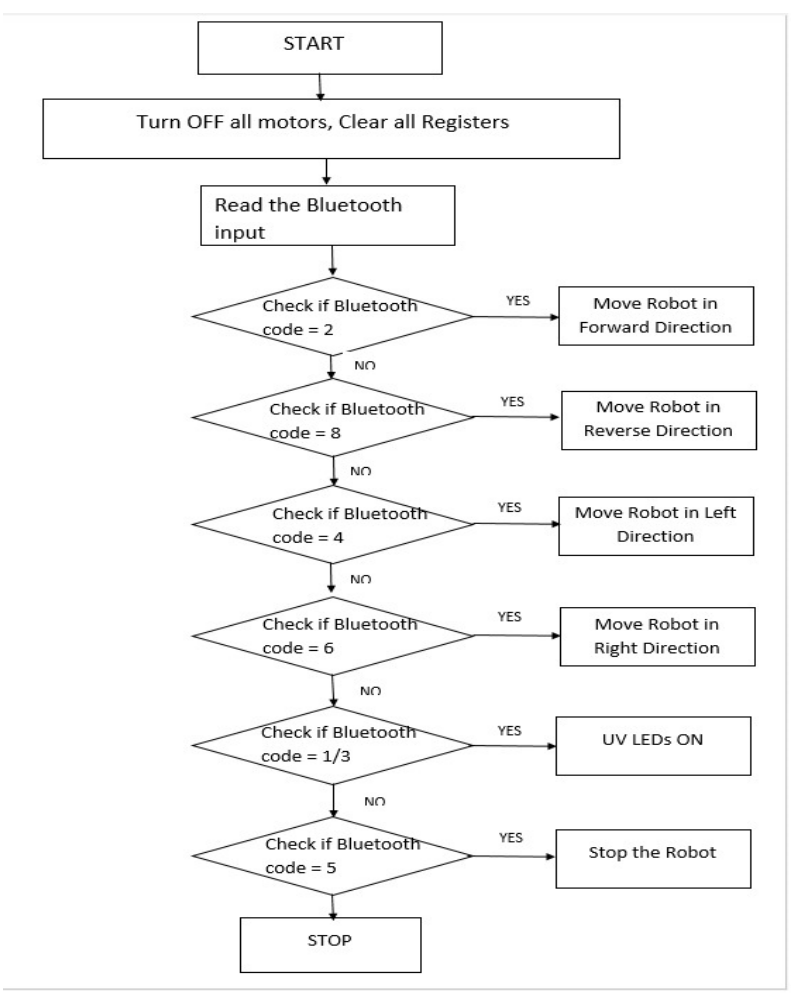

Fig. 2. Flowchart of the Proposed System

\section{Algorithm}

Once the robot is switched $\mathrm{ON}$, the connection is established between android and Bluetooth decoder. The microcontroller will check the input of Bluetooth decoder. If the input of the decoder is 2 , robot will move in forward direction. If Bluetooth decoder input is 8 , robot moves in reverse. For 4 in left and 6 in right direction. Apart from movement of robot, the microcontroller also receives signal from Bluetooth decoder for switching ON/OFF of UVC LEDs. If the input of the decoder is 1 or 3 , it will switch on the LEDs. If microcontroller receives 5 as an input, it will switch off the LEDs and stop the robot.

\section{RESUlt AND Discussion}

The robot is suitable for any type of floor and is operated by Bluetooth technology. The motors consumes less power, so it will be energy saving and cost savings too. The harmful micro-organisms present on the floor is destroyed by Ultraviolet LED. The UV LED destroys the DNA/RNA of the microorganisms using the powerful UV rays. This is an ecofriendly method as UV light does not leave any residue. It is very essential to design the device in way that UV-C light will not affect the humans or its rays will not come in the eyes of users. Here, we have developed the design that follow the guidelines of using UV-C light and it will not affect the humans as it is very dangerous to direct exposure [6].

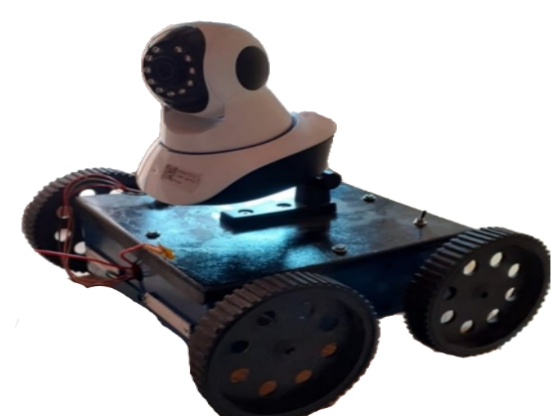

Fig. 3. Working model of UV Sanitization Robot

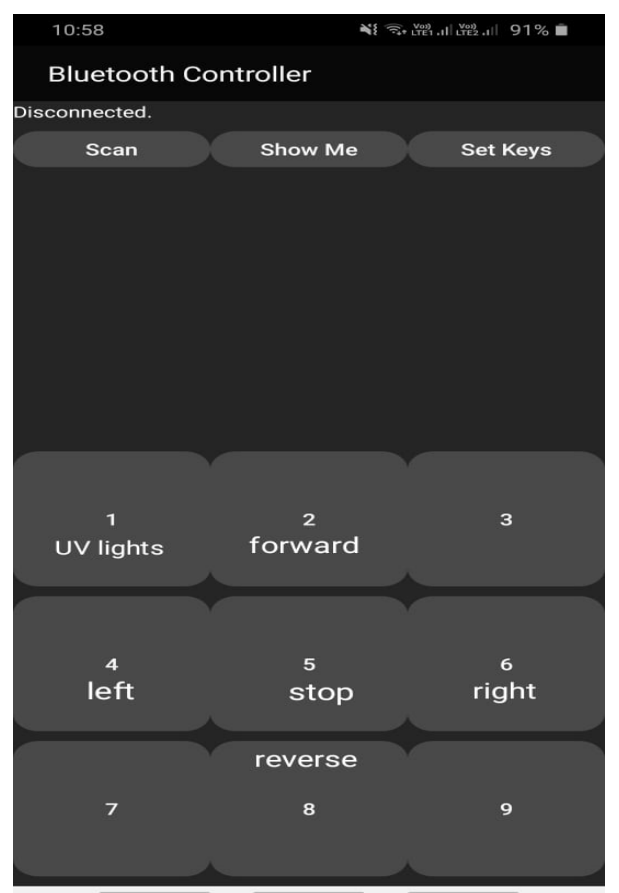

Fig. 4. Control Interface 


\section{Conclusion}

The creation of clinical robotics has appreciably increased the safety and standard of health control systems due to Healthcare digitization.Thus, we have developed a sanitization device to perform disinfection of objects, floor etc. Ultraviolet sanitization is the latest disinfection technology with increasing importance. It can be used in clinics and other commercial purposes to improve the way of life. The main benefit of this project is to support health workers. The future scope for this project can be making this robot autonomously driven so that even less human intervention is required and making the robot base more resilient to rough terrain for smoother operation.

\section{REFERENCES}

[1] Pacharawam Chanprakon, Tapparat Sae-Oung, Treesukon Treebupachatsakul "An Ultra-violet sterilization robot for disinfection" IEEE Xplore, August 19.

[2] Himadri Nath Saha, Sourav Gon, Annesha Nayak, Samabrita kundu, Sumandrita Moitra, "IoT Based Garbage Monitoring and Clearance Alert System" 2018 IEEE 9th Annual Information Technology, Electronics and Mobile Communication Conference (IEMCON) Pages: $204-208$.

[3] Noriyuki Yagi, Mirei Mori, Akiko Hamamoto, Masayuki Nakano, Masatake Akutagawa, "Sterilization Using 365 Nm Uv-Led", 29th Annual International Conference of the IEEE EMBS Cité Internationale 2007. Karthick.T, Ravikumar.A, Selvakumar.L, Viknesh.T, Parthiban.B Gopinath.A. "Simple Autonomous Cleaner Robot". International Journal of Pure and Applied Mathematics, Volume 118 No. 202018.

[4] Sivaranjani, Mayalvizhi, Swathy, Venkatesan, Janarth, "DESIGN OF A PORTABLE ASSISTIVE DEVICE FOR DISINFECTION OF FLOOR USING BLUETOOTH TECHNOLOGY". International Journal of Science,Engineering and Technology Research(IJSETR), Volume 4,Issue-5,May 2015.

[5] G Tuangzhi Dai and Tiequn Chen,"Design on measurement and control system of cleaning robot based on sensor array detetion",In IEEE International conference on control automation Guangzhou, 30 to June $1,2015$.

[6] Sahil Parmar, Hardik Bhatt, "A DESIGN OF HYBRID FLOOR UV-C STERILIZER ROBOT',International Research Journal of Engineering and Technology (IRJET), Gujarat, India.

[7] Apeksha Wadibhasme, Yedhubooshan M M, Kaushik Moolya, Shireen Farhath, Dipti Darade, Sumana Hati, "SANITIZATION ROBOT"..International Research Journal of Engineering and Technology (IRJET), Volume 7, Issue 8, Aug 2020.

[8] Thomas Rubaek, Merima Cikotic, Simon Falden, "Evaluation Of The Uv-Disinfection Robot”, 2016. 